\title{
Validation of simultaneous deregression of cow and bull breeding values and derivation of appropriate weights
}

\author{
M. P. L. Calus, ${ }^{1}$ J. Vandenplas, J. ten Napel, and R. F. Veerkamp \\ Animal Breeding and Genomics Centre, Wageningen UR Livestock Research, $6700 \mathrm{AH}$ Wageningen, the Netherlands
}

\begin{abstract}
Training of genomic prediction in dairy cattle may use deregressed proofs (DRP) as phenotypes. In this case, DRP should be estimated breeding values (EBV) corrected for information of relatives included in the data used for genomic prediction, and adjusted for regression to the mean (i.e., their reliability). Deregression is especially important when combining animals with EBV with low reliability, as commonly the case for cows, and high reliability. The objective of this paper, therefore, was to compare the performance of different deregression procedures for data that include both cow and bull EBV, and to develop and test procedures to obtain the appropriate deregressed weights for the DRP. Considered DRP were EBV: without any adjustment, adjusted for information of parents and regression to the mean, or adjusted for information of all relatives and regression to the mean. Considered deregressed weights were weights of initial EBV: without any adjustment, adjusted for information of parents, or adjusted for information of all relatives. The procedures were compared using simulated data based on an existing pedigree with 1,532 bulls and 13,720 cows that were considered to be included in the data used for genomic prediction. For each cow, 1 to 5 records were simulated. For each bull, an additional 50 to 200 daughters with 1 record each were simulated to generate a source of data that was not used for genomic prediction. The simulated trait had either a heritability of 0.05 or 0.3 . The validation involved 3 steps: (1) computation of initial EBV and weights, (2) deregression of those EBV and weights, (3) using deregressed EBV and weights to compute final EBV, (4) comparison of the initial and final EBV and weights. The methods developed to compute appropriate weights for the DRP were either very precise and computationally somewhat demanding for larger data sets, or were less precise but computationally trivial due their approximate nature. Adjusting DRP for all relatives, known as matrix
\end{abstract}

Received February 12, 2016.

Accepted April 6, 2016.

${ }^{1}$ Corresponding author: mario.calus@wur.nl deregression, yields by definition final EBV that are identical to the original EBV. Matrix deregression is therefore preferred over other approaches that only correct for information of parents or not performing any deregression at all. It is important to use appropriate weights for the DRP, properly corrected for information of relatives, especially when individual reliabilities of final EBV are computed based on the prediction error variance of the model.

Key words: deregression, deregressed proofs, reference population

\section{INTRODUCTION}

Several applications on dairy cattle data use pseudophenotypes as response variables, such as international genetic evaluations performed by Interbull (Schaeffer, 1994), integration of foreign information into a national genetic evaluation (e.g., Banos et al., 1992), QTL mapping (e.g., Rodriguez-Zas et al., 2002; Boichard et al., 2003), and genomic prediction (Meuwissen et al., 2001). The pseudo-phenotypes usually summarize the phenotypic performance of female progeny of bulls. They can also summarize the phenotypic performance of individual dairy cows, for instance across lactations. Commonly used pseudo-phenotypes for cows and bulls are so-called yield deviations (YD) and daughter yield deviations (VanRaden and Wiggans, 1991). Those YD are derived from a model that uses raw phenotypic data as input and adjusts for fixed effects such as herd-yearseason. For many applications, YD cannot be derived, because the raw phenotypic data are not available. In addition, the analysis of certain traits requires complicated models, such as survival analysis using a proportional hazards model (Ducrocq, 1994), which makes derivation of YD difficult. In those cases where YD are not available, information of EBV, commonly derived from national genetic evaluations, are used to compute pseudo-phenotypes (e.g., Rozzi et al., 1990; Banos et al., 1993; Bonaiti and Boichard, 1995; Jairath et al., 1998).

Breeding values are commonly estimated using BLUP (Henderson, 1949). One of the features of BLUP is that it regresses EBV to the mean to avoid bias in 
the prediction of phenotypes. This feature makes the variance of EBV proportional to their reliability, and the higher the reliability, the higher the variance of the EBV. For the aforementioned applications that use pseudo-phenotypes as response variables, it is required that the pseudo-phenotypes give the same EBV and reliabilities as obtained from the underlying genetic evaluation. This is commonly achieved by deregressing the EBV. The most simple form of deregression involves dividing each EBV by its reliability (Goddard, 1985; Garrick et al., 2009). The resulting variables are commonly termed deregressed proofs (DRP).

Apart from the difference in variance of EBV with different reliabilities, an additional issue is that EBV include information of related animals whose EBV will also be used in the subsequent analyses. Nevertheless, the subsequent analyses assume that the pseudo-phenotypes derived from the EBV are measured on the animal itself, and this information is then propagated toward relatives in the same subset. To avoid issues due to double counting of the same information, information that comes from relatives that are included with their data in the subsequent analyses should be removed from the pseudo-phenotypes. Sophisticated deregression procedures enable the simultaneously adjusting of EBV for differences in reliabilities as well as to account for information of relatives (e.g., Sigurdsson and Banos, 1995; Jairath et al., 1998). For each DRP value, a weight, such as the number of effective daughter contributions (EDC; Fikse and Banos, 2001), is commonly computed that reflects its reliability. When DRP are corrected for information of relatives, this also means that appropriate weights of the DRP are on average expected to be lower than the corresponding weights of the original EBV because the DRP contain less information than the EBV.

Whether or not deregression of EBV and computation of appropriate weights has a (large) effect on the results in subsequent analyses depends on differences between reliabilities of EBV across animals due to different amounts of information per individual. In dairy cattle, typically a few influential bulls have many progeny in the population, whereas a large number of bulls each have a limited number of progeny. Nevertheless, bulls with proofs based on daughter performance generally have reliabilities that are greater than 0.5 . In these cases, the amount of "own" information is relatively large, and the effect of accounting (or not) for information of relatives in the deregression procedure is expected to have relatively little effect (Sigurdsson and Banos, 1995; Guo et al., 2010). When animals with substantially lower reliabilities are included, such as cows, the effect of deregression is expected to be much more important. For example, Vandenplas et al. (2014b) estimated that $>85 \%$ of the total information of animals with a reliability lower than 0.50 for milk traits was due to information from relatives in the data, whereas this percentage was lower than $7 \%$ for dairy bulls having daughters with records. In the context of genomic prediction, deregression of EBV with low reliability that are heavily influenced by information of relatives is expected to become more important in the near future. This follows directly from the observation that reference populations, which currently mainly include genotyped bulls with proofs based on daughter information (VanRaden et al., 2009; Lund et al., 2011), will be further expanded by adding genotyped cows, both for conventional (Mc Hugh et al., 2011; Lourenco et al., 2014; Koivula et al., 2016) and new breeding goal traits (Buch et al., 2012; Calus et al., 2013; EggerDanner et al., 2014).

The objective of this paper, therefore, was to compare the performance of different deregression procedures for data that includes both cow and bull EBV, and to develop and test procedures to obtain the appropriate deregressed weights that should be used with those DRP. In this paper, we refer to a situation in which EBV of animals included in a reference population for genomic prediction need to be deregressed, to be used as pseudo-phenotypes in a subsequent genomic prediction model, but the described methods are also applicable for other situations.

\section{MATERIALS AND METHODS}

\section{Deregression of EBV}

Deregression is a procedure that computes pseudophenotypes, here referred to as DRP, that will yield a set of currently known EBV in a BLUP evaluation. The procedure used to compute DRP will be referred to as matrix deregression. Consider that we want to deregress a set of EBV of animals included in a reference population, and that this set of animals is a subset of a larger data set used in an evaluation system to obtain the EBV. In this case, the deregression procedure should correct within the subset all EBV for information of relatives within this subset. For instance, the EBV of a sire that is based on 100 daughters, of which 10 are genotyped and also included in the reference population, should be corrected for those 10 genotyped daughters, such that the DRP still includes the information of the 90 daughters that are not genotyped and therefore not included in the reference population. The same applies for the weight given to the DRP, as this should reflect only the information of the 90 daughters not included in the reference population. The matrix deregression procedure (following e.g., Jairath et al., 
1998) involves setting up the following mixed model equations (MME) for a pedigree-based model that includes all animals in the subset (i.e., the animals included in the reference population in our case) and their ancestors:

$$
\left[\begin{array}{ccc}
\mathbf{1}^{\prime} \mathbf{R}^{-1} \mathbf{1} & \mathbf{1}^{\prime} \mathbf{R}^{-1} \mathbf{Z} & \mathbf{0} \\
\mathbf{Z}^{\prime} \mathbf{R}^{-1} \mathbf{1} & \mathbf{Z}^{\prime} \mathbf{R}^{-1} \mathbf{Z}+\mathbf{A}^{\mathrm{gg}} \lambda & \mathbf{A}^{\mathrm{gu}} \lambda \\
\mathbf{0} & \mathbf{A}^{\mathrm{ug}} \lambda & \mathbf{A}^{\mathrm{uu}} \lambda
\end{array}\right]\left[\begin{array}{c}
\hat{\mu} \\
\hat{\mathbf{a}}_{\mathrm{g}} \\
\hat{\mathbf{a}}_{\mathrm{u}}
\end{array}\right]=\left[\begin{array}{c}
\mathbf{1}^{\prime} \mathbf{R}^{-1} \mathbf{y} \\
\mathbf{Z}^{\prime} \mathbf{R}^{-1} \mathbf{y} \\
\mathbf{0}
\end{array}\right]
$$

where $\mathbf{1}$ is a vector of ones, $\mathbf{Z}$ is an incidence matrix for random additive genetic effects (note that $\mathbf{Z}$ is an identity matrix in this case and can therefore be omitted), $\mathbf{R}^{-1}$ is assumed to be a diagonal matrix with diagonal elements equal to weights of the DRP, and $\mathbf{A}^{-1}$ is the inverse relationship matrix including all animals whose EBV should be deregressed and their full pedigree. The matrix $\mathbf{A}^{-1}$ is partitioned as follows:

$$
\mathbf{A}^{-1}=\left[\begin{array}{ll}
\mathbf{A}^{\mathrm{gg}} & \mathbf{A}^{\mathrm{gu}} \\
\mathbf{A}^{\mathrm{ug}} & \mathbf{A}^{\mathrm{uu}}
\end{array}\right],
$$

where $\mathbf{A}^{\text {gg }}$ is the part of $\mathbf{A}^{-1}$ for the genotyped animals in the reference population, $\mathbf{A}^{\mathrm{uu}}$ is the part of $\mathbf{A}^{-1}$ for ungenotyped animals, and $\mathbf{A}^{\mathrm{ug}}$ is the off-diagonal part of $\mathbf{A}^{-1}$ between genotyped and ungenotyped animals. Finally, $\lambda=\frac{\sigma_{e}^{2}}{\sigma_{a}^{2}}, \hat{\mu}$ is the overall mean, $\hat{\mathbf{a}}_{\mathrm{g}}$ is a vector of EBV of the genotyped animals that needs to be deregressed, $\hat{\mathbf{a}}_{\mathrm{u}}$ is a vector of EBV of ungenotyped animals that need to be computed solely from $\hat{\mathbf{a}}_{\mathrm{g}}$, and $\mathbf{y}$ is a vector of DRP that need to be computed. Note that the mean can be absorbed in the EBV (Lien et al., 1995), or omitted from the above (e.g., Sigurdsson and Banos, 1995; Harris and Johnson, 2010; Ricard et al., 2013). However, not accounting for the mean may lead to inconsistent results for traits with a low heritability (Rogers et al., 1996; Jairath et al., 1998). Therefore, we performed matrix deregression that accounts for the mean as outlined by Jairath et al. (1998). This involves computing the DRP and $\mu$ iteratively. Based on MME [1], this was done as follows:

1. Set $\hat{\mu}$ to zero.

2. Compute $\hat{\mathbf{a}}_{\mathrm{g}}=\widehat{\mathbf{E B V}}-\hat{\mu}$ where $\widehat{\mathbf{E B V}}$ stores the original EBV.

3. Compute $\hat{\mathbf{a}}_{\mathrm{u}}=-\left(\mathbf{A}^{\mathrm{uu}}\right)^{-1} \mathbf{A}^{\mathrm{ug}} \hat{\mathbf{a}}_{\mathrm{g}}$.

4. Compute $\mathbf{R}^{-1} \mathbf{y}=\left[\begin{array}{lll}\mathbf{R}^{-1} \mathbf{1} & \left(\mathbf{R}^{-1}+\mathbf{A}^{g g} \lambda\right) & \mathbf{A}^{g u} \lambda\end{array}\right]\left[\begin{array}{c}\hat{\mu} \\ \hat{\mathbf{a}}_{\mathrm{g}} \\ \hat{\mathbf{a}}_{\mathrm{u}}\end{array}\right]$.
5. Compute $\hat{\mu}=\left(\mathbf{1}^{\prime} \mathbf{R}^{-1} \mathbf{1}\right)^{-1} \mathbf{1}^{\prime} \mathbf{R}^{-1} \mathbf{y}$.

6. Iterate 2 to 5 until convergence.

7. Compute the DRP as $\mathbf{D R P}=\mathbf{R R}^{-1} \mathbf{y}$.

Step 3 involves computation of EBV of all nongenotyped animals in the pedigree, which can be extended to include genetic groups as described by Jairath et al. (1998), conditional on the EBV of the genotyped reference animals that are to be deregressed. As pointed out by Fernando et al. (2014), for a similar scenario where genotypes are to be predicted instead of EBV, $\hat{\mathbf{a}}_{\mathrm{u}}$ can efficiently be computed by solving the following very sparse system:

$$
\mathbf{A}^{\mathrm{uu}} \hat{\mathbf{a}}_{\mathrm{u}}=-\mathbf{A}^{\mathrm{ug}} \hat{\mathbf{a}}_{\mathrm{g}} .
$$

Solving this system was done using a conjugate gradient algorithm for solving $x$ in a linear system $A x=b$, with $A=A^{u u}, x=\hat{a}_{u}$, and $b=-A^{u g} \hat{a}_{g}$ (Shewchuck, 1994). Step 5 computes a new value for $\hat{\mu}$, conditional on the DRP that are updated in step 4 . The convergence criterion used in step 6 was computed in iteration $l$ as

$$
\frac{\sum_{k=1}^{n}\left(\mathrm{DRP}_{k}^{l}-\mathrm{DRP}_{k}^{l-1}\right)^{2}}{\sum_{i=1}^{n}\left(\mathrm{DRP}_{k}^{l}\right)^{2}},
$$

where $\mathrm{DRP}_{k}^{l}$ is the DRP of animal $k$ in iteration $l$. Convergence was assumed once the value of this convergence criterion dropped below $10^{-8}$. The DRP computed as described above will hereafter be referred to as DRP_mat.

\section{Deregression of Weights}

The matrix deregression procedure to compute DRP requires weights, included in the diagonal matrix $\mathbf{R}^{-1}$ in MME [1], that reflect the amount of information retained in the DRP, or in other words, the amount of information of an animal in its DRP coming from own records or from relatives outside the reference population. The starting point to calculate those deregressed weights is the weights used to compute the original EBV. These weights are here referred to as effective record contributions (ERC; e.g., Prribyl et al., 2013), also being termed the effective number of records (Misztal and Wiggans, 1988), and computed as

$$
\mathrm{ERC}_{k}=\lambda \frac{\mathrm{REL}_{k}}{1-\mathrm{REL}_{k}}
$$


where $\mathrm{REL}_{k}$ is the reliability of the EBV of animal $k$. Note that ERC are used instead of the more commonly used EDC (Fikse and Banos, 2001), because the model described in Equation [1] is an animal model. However, EDC could easily be used instead and involves computing $\lambda$ for a sire instead of for an animal model, that is, as $\lambda_{s}=\frac{\sigma_{e}^{2}+\frac{3}{4} \sigma_{a}^{2}}{\frac{1}{4} \sigma_{a}^{2}}=\frac{4-h^{2}}{h^{2}}$, and then computing EDC as

$$
\mathrm{EDC}_{k}=\lambda_{s} \frac{\mathrm{REL}_{k}}{1-\mathrm{REL}_{k}}=\frac{\lambda_{s}}{\lambda} \mathrm{ERC}_{k}
$$

Here we developed a procedure to compute dERC (i.e., deregressed ERC), that simultaneously adjusts an animal's ERC for information from all its genotyped relatives with phenotypic information in the data. This is achieved by iteratively computing the part of the ERC that comes from genotyped relatives in the reference population $\left(\mathrm{ERC}_{\mathrm{RP}_{k}}\right)$, and then the dERC of animal $k$ is computed as

$$
\mathrm{dERC}_{k}=\mathrm{ERC}_{k}-\mathrm{ERC}_{\mathrm{RP}_{k}} .
$$

The procedure starts with initializing dERC values by setting them equal to the ERC values for all animals (note that any other value, e.g., setting all ERC values to zero, yields the same result). Thereafter, the following steps are taken for each animal:

1. Set up and invert the left-hand side (LHS) of MME of a model that computes EBV from the DRP, such as shown below in Equation [2], using current dERC values as weights in the diagonal matrix $\mathbf{R}^{-1}$. This LHS is stored as LHS_0;

2 . For each animal $k$, one animal at the time, remove its current $\mathrm{dERC}_{k}$ from LHS_0 to obtain LHS_0*, such that in LHS_0* effectively dERC ${ }_{k}$ $=0$. Then, directly compute (and store) $\mathrm{ERC}_{\mathrm{RP}_{k}}$ from LHS_0*;

3. Using the $\mathrm{ERC}_{\mathrm{RP}}$ values for all animals in step 2, computeforeachanimaldERC $\mathrm{k}_{\mathrm{k}}=\mathrm{ERC}_{\mathrm{k}}-\mathrm{ERC}_{\mathrm{RP}_{k}}$ to obtain updated dERC values for all animals;

4. Repeat steps 1 to 3 until convergence.

Step 1 is based on the following MME, that are equivalent to those used in the approach of Ricard et al. (2013):

$$
\left[\begin{array}{cc}
\mathbf{1}^{\prime} \mathbf{R}^{-1} \mathbf{1} & \mathbf{1}^{\prime} \mathbf{R}^{-1} \mathbf{Z} \\
\mathbf{1}^{\prime} \mathbf{R}^{-1} \mathbf{Z} & \mathbf{Z}^{\prime} \mathbf{R}^{-1} \mathbf{Z}+\mathbf{A}_{\mathrm{gg}}^{-1} \lambda
\end{array}\right]\left[\begin{array}{c}
\hat{\mu} \\
\hat{\mathbf{a}}_{\mathrm{g}}
\end{array}\right]=\left[\begin{array}{c}
\mathbf{1}^{\prime} \mathbf{R}^{-1} \mathbf{y} \\
\mathbf{Z}^{\prime} \mathbf{R}^{-1} \mathbf{y}
\end{array}\right]
$$

that is equivalent to [1], after the absorption of the 3rd equation of the MME [1], to reduce the size of the LHS. The MME [2] requires an inverse pedigree-based relationship matrix including only animals in the reference population $\left(\mathbf{A}_{\mathrm{gg}}^{-1}\right)$. Therefore, the full numerator relationship matrix for all genotyped reference animals with $\operatorname{EBV}\left(\mathbf{A}_{g g}\right)$ was computed using the full pedigree and considering inbreeding coefficients, and inverted to obtain $\mathbf{A}_{\mathrm{gg}}^{-1}$. In step 2, the adjusted inverse LHS was obtained using the inverted LHS obtained in step 1. Realizing that the only difference between the 2 LHS matrices is a change of one diagonal value, the procedure outlined by Hager (1989) can be used. Consider that we have an $n \times n$ square invertible matrix LHS (the full LHS) and a matrix LHSR that is identical to LHS except for the elements in one column, being the diagonal element for animal $k$ in our case, then the element $\operatorname{lhsr}_{k k}^{-1}$ of $\mathbf{L H S R} \mathbf{R}^{-1}$ can be expressed in terms of elements of $\mathbf{L H S}^{-1}$ and $\mathbf{L H S R}$ as

$$
\operatorname{lhsr}_{k k}^{-1}=\frac{\operatorname{lhs}_{k k}^{-1}}{\sum_{i=1}^{n} \operatorname{lhs}_{k i}^{-1} \operatorname{lhsr}_{i k}} .
$$

Then, the final part of step 2 involved computing $\mathrm{ERC}_{\mathrm{RP}_{k}}$ directly as

$$
\mathrm{ERC}_{\mathrm{RP}_{k}}=\frac{1-\operatorname{lhsr}_{k k}^{-1} \lambda}{\operatorname{lhsr}_{k k}^{-1}} .
$$

Using the above modification of the inverse of the LHS for each animal implies that for the above algorithm the LHS of the MME [2] needs to be inverted only once per iteration, whereas the other computations per iteration are trivial. Nevertheless, inverting $\mathbf{A}_{\mathrm{gg}}$ once and the full LHS multiple times becomes computationally quite demanding with increasing numbers of animals, and may therefore be prohibitive in practical applications. To reduce the computational burden of the algorithm to compute the dERC values, denoted as $\mathrm{dERC}_{\mathrm{LHS}}$, matrix inversion was performed using LAPACK (Anderson et al., 1990), and efficient algorithms were used for the computation of the inbreeding coefficients (Sargolzaei et al., 2005) and the full numerator relationship matrix $\mathbf{A}_{\mathrm{gg}}$ (Colleau, 2002). A few things should be noted on the procedure described above. First, a similar algorithm has been described to be used in the context 
of integrating external information into genetic evaluations (Vandenplas and Gengler, 2012). Second, this method considers prediction error co-variances, but not explicitly those that arise due to original fixed effects structures such as contemporary groups, resulting in them being ignored when performing the deregression.

The convergence criterion used in step 4 was computed in iteration $l$ as

$$
\frac{\sum_{k=1}^{n}\left(\mathrm{dERC}_{\mathrm{LHS}, k}^{l}-\mathrm{dERC}_{\mathrm{LHS}, k}^{l-1}\right)^{2}}{\sum_{k=1}^{n}\left(\mathrm{dERC}_{\mathrm{LHS}, k}^{l}\right)^{2}},
$$

and convergence was assumed once its value dropped below $10^{-28}$. A maximum of 5 iterations was imposed because preliminary analyses showed that this was generally sufficient. The dERC computed as described above will hereafter be referred to as dERC_LHS.

Other procedures that are used to disentangle ERC or reliabilities due to different sources of information usually avoid setting up and inverting the LHS of the corresponding MME, and use selection index theory instead. This can either be achieved at the level of reliabilities using the information source method (Harris and Johnson, 1998; Ducrocq and Liu, 2009; Harris and Johnson, 2010), or by iteratively computing ERC due to various sources of information. Following these methods, we used an iterative procedure that involves going up and down through the pedigree, similar to the method presented by Harris and Johnson (2010). This method starts with the reliability of an animals EBV, $\mathrm{REL}_{k}(\mathrm{EBV})$, and involves iteratively removing $\mathrm{REL}_{\mathrm{RP}_{k}}$ coming from genotyped relatives in the data, notably from progeny and parents, to obtain a deregressed reliability (i.e., $\mathrm{dREL}_{k}$ ), which can be converted to $\mathrm{dERC}_{k}$, using the following steps:

1. Initialize for all animals $\mathrm{dREL}_{k}=\mathrm{REL}_{k}(\mathrm{EBV})$.

2 . From the youngest to the oldest animal $k$; compute its contribution to the reliability of any genotyped parent, for instance denoted as $\operatorname{REL}_{j}(p)$ for considering the current values of $\mathrm{dREL}_{k}$ and $\operatorname{REL}_{k}(p)$.

3. From the oldest to the youngest animal $k$ :

a. Compute the reliability due to information of the parents, denoted as $\operatorname{REL}_{k}(p a)$, considering any parent $j$, and

b. Compute the updated $\mathrm{dREL}_{k}$ by subtracting the reliability due to progeny and parents from an animal's initial reliability.
4. Repeat 2 and 3 until convergence.

5. Convert $\mathrm{dREL}_{k}$ to $\mathrm{dERC}_{k}$.

Step 2 involves looping through all genotyped animals, denoted as $k$, from youngest to oldest, to compute the updated reliability of parent $j$ due to progeny [i.e., $\left.\operatorname{REL}_{j}^{\prime}(p)\right]$ using the information source method (Harris and Johnson, 1998) as

$$
\begin{aligned}
& \operatorname{REL}_{j}^{\prime}(p)= \\
& \frac{\operatorname{REL}_{j}(p)+x_{k j}^{2} \operatorname{REL}_{k}(o+p)-2 x_{k j}^{2} \operatorname{REL}_{j}(p) \operatorname{REL}_{k}(o+p)}{1-\operatorname{REL}_{j}(p) x_{k j}^{2} \operatorname{REL}_{k}(o+p)},
\end{aligned}
$$

where $\mathrm{REL}_{k}(o+p)$ is the reliability of progeny $k$ of animal $j$ due to its own performance $(o)$ and its progeny $(p)$, and $x_{k j}^{2}=0.25$ when ignoring inbreeding. $\operatorname{REL}_{k}(o$ $+p)$ is calculated as

$$
\operatorname{REL}_{k}(o+p)=\frac{\mathrm{dREL}_{k}+\operatorname{REL}_{k}(p)-2 \mathrm{dREL}_{k} \operatorname{REL}_{k}(p)}{1-\operatorname{dREL}_{k} \operatorname{REL}_{k}(p)} .
$$

Because this step loops from youngest to oldest, $\operatorname{REL}(p)$ values of progeny are always computed before their parents, and therefore included in $\operatorname{REL}(o)$ values of their parents.

Step 3a involves computing the reliability of animal $k$ due to any of its parents included in the reference population. This first involves computing the reliability for any parents included in the reference population, of which the reliability of animal $k$ based on own performance and its progeny is removed. For instance, for the sire of animal $k$, this reliability will be denoted as $\mathrm{REL}_{\text {sire }_{k}}(\mathrm{EBV} \mid k)$, and is computed as

$$
\begin{aligned}
& \mathrm{REL}_{\text {sire }_{k}}(\mathrm{EBV} \mid k)= \\
& \frac{\mathrm{REL}_{\text {sire }_{k}}(\mathrm{EBV})-x_{k s}^{2} \mathrm{REL}_{k}(o+p)}{\mathrm{REL}_{\text {sire }_{k}}(\mathrm{EBV}) x_{k s}^{2} \mathrm{REL}_{k}(o+p)+1-2 \mathrm{REL}_{k}(o+p)},
\end{aligned}
$$

and $\mathrm{REL}_{\mathrm{dam}_{k}}(\mathrm{EBV} \mid k)$ is computed in the same way. Then, the reliability for animal $k$ due to its parents is computed as

$$
\operatorname{REL}_{k}(\mathrm{pa})=x_{k s}^{2} \mathrm{REL}_{\mathrm{sir}_{k}}(\mathrm{EBV} \mid k)+x_{k d}^{2} \mathrm{REL}_{\mathrm{dam}_{k}}(\mathrm{EBV} \mid k),
$$

where $x_{k s}^{2}$ and $x_{k d}^{2}$ are 0.25 when ignoring inbreeding.

Step $3 \mathrm{~b}$ involves computation of the reliability of each animal $k$ due to information of all relatives, i.e., 
progeny and parents, in the reference population (RP) as

$\operatorname{REL}_{k}(\mathrm{RP})=\frac{\operatorname{REL}_{k}(p)+\mathrm{REL}_{k}(\mathrm{pa})-2 \mathrm{REL}_{k}(p) \mathrm{REL}_{i}(\mathrm{pa})}{1-\mathrm{REL}_{k}(p) \operatorname{REL}_{k}(p a)}$,

And using this to finally compute $\mathrm{dREL}_{k}$ as

$$
\mathrm{dREL}_{k}=\frac{\mathrm{REL}_{k}(\mathrm{EBV})-\mathrm{REL}_{k}(\mathrm{RP})}{\mathrm{REL}_{k}(\mathrm{EBV}) \mathrm{REL}_{k}(\mathrm{RP})+1-2 \mathrm{REL}_{k}(\mathrm{RP})} .
$$

Convergence in step 4 was assessed in a similar way as for the computation of $\mathrm{dERC}_{\mathrm{LHS}}$ values, and the procedure was stopped if the convergence criterion was not met after 100 iterations. Finally, step 5 involves computing deregressed $\mathrm{ERC}$ values (i.e., $\mathrm{dERC}_{k}$ ), as

$$
\mathrm{dERC}_{k}=\lambda \frac{\mathrm{dREL}_{k}}{1-\mathrm{dREL}_{k}} .
$$

The dERC obtained with this iterative procedure will hereafter be referred to as dERC_iter, as it uses the iterative process described above to approximate dERC_LHS. Computation of the dERC_iter is similar to approaches used by others (e.g., Harris and Johnson, 2010; Ricard et al., 2013), which usually ignore inbreeding. Because the procedure outlined above aims to compute a value equivalent to $\mathrm{dEDC}_{\mathrm{lhs}}$ where inbreeding was included when setting up $\mathbf{A}^{-1}$ to compute the $\mathrm{EBV}$, we extended the iterative procedure to include inbreeding coefficients as well. Considering inbreeding, the values for $x$ were computed as follows. To compute the contribution to the reliability of the EBV of an animal $k\left(a_{k}\right)$ by the EBV of one of its parents [e.g., the EBV of its sire $s\left(a_{s}\right)$ ], the correlation between their EBV is required. This correlation, here termed $x_{k s}$, is computed as

$$
\begin{aligned}
& x_{k s}=\frac{\sigma\left(a_{k}, a_{s}\right)}{\sqrt{\operatorname{Var}\left(a_{k}\right) \operatorname{Var}\left(a_{s}\right)}}=\frac{\sigma\left(\frac{1}{2} a_{s}+\frac{1}{2} a_{d}+M S_{k}, a_{s}\right)}{\sqrt{\operatorname{Var}\left(a_{k}\right) \operatorname{Var}\left(a_{s}\right)}}= \\
& \frac{\frac{1}{2}\left(1+F_{s}\right) \sigma_{a}^{2}+\frac{1}{2} \sigma\left(a_{s}, a_{d}\right)}{\sigma_{a}^{2} \sqrt{1+F_{k}} \sqrt{1+F_{s}}}=\frac{\frac{1}{2}\left(1+F_{s}\right) \sigma_{a}^{2}+F_{k} \sigma_{a}^{2}}{\sigma_{a}^{2} \sqrt{1+F_{k}} \sqrt{1+F_{s}}}= \\
& \frac{\frac{1}{2}\left(1+F_{s}\right)+F_{k}}{\sqrt{1+F_{k}} \sqrt{1+F_{s}}}
\end{aligned}
$$

where $\sigma\left(a_{k}, a_{s}\right)$ is the covariance between $a_{k}$ and $a_{s}, \sigma_{a}^{2}$ is the additive genetic variance as before, $\mathrm{MS}_{k}$ is the
Mendelian sampling term of animal $k$, and $F_{s}$ and $F_{k}$ are the inbreeding coefficients of the parent $s$ and animal $k$. From this, it directly follows that $x_{k s}^{2}=0.25$ when ignoring inbreeding. The obtained dERC, considering inbreeding, will hereafter be referred to as dERC_ iter_F.

For comparison, the results obtained with the above described dERC values were compared with the use of the ERC of the EBV without deregression, and to the use of ERC values after removing information of genotyped parents, following the procedure presented by VanRaden et al. (2009):

$$
\mathrm{dERC}_{k}=\mathrm{ERC}-\mathrm{ERC}_{k}(\mathrm{pa}),
$$

where $\mathrm{ERC}_{k}(\mathrm{pa})$ is computed from the reliability of the parent average $\mathrm{EBV}, \mathrm{REL}_{k}(\mathrm{pa})$, which is computed as $\mathrm{REL}_{k}(\mathrm{pa})=\frac{1}{4}\left(\mathrm{REL}_{\text {sire }_{k}}+\mathrm{REL}_{\mathrm{dam}_{k}}\right)$. This dERC value will hereafter be referred to as dERC_VR.

For comparison, the results obtained from matrix deregression were compared with the use of EBV without deregression, and to the use of DRP obtained with the procedure outlined by VanRaden et al. (2009) and Wiggans et al. (2011). Briefly, this method computes DRP as follows:

$$
\mathrm{DRP}_{k}=\mathrm{PA}_{k}+\left(\mathrm{EBV}_{k}-\mathrm{PA}_{k}\right)\left[\frac{\mathrm{ERC}_{k}}{\mathrm{dERC}_{k}}\right],
$$

where $\mathrm{PA}_{k}$ is the average of the EBV of the parents of animal $k, \mathrm{ERC}_{k}$ is the ERC that belongs to $\mathrm{EBV}_{k}$, and $\mathrm{dERC}_{k}$ is the deregressed ERC for animal $k$, as discussed previously. Note that VanRaden et al. (2009) used EDC instead of ERC values, but the computed $\mathrm{DRP}_{k}$ is not different when using one or the other because ERC can simply be obtained as a linear transformation of EDC. This DRP value will hereafter be referred to as DRP_VR. A similar but slightly different version of the above follows from the deregression proposed by Garrick et al. (2009):

$$
\mathrm{DRP}_{k}=\mathrm{PA}_{k}+\frac{\left(\mathrm{EBV}_{k}-\mathrm{PA}_{k}\right)}{\mathrm{REL}_{k}(o+p)},
$$

where $\operatorname{REL}_{k}(o+p)$, as before, is the reliability of animal $k$ due to its own performance (o) and its progeny $(p)$. This DRP value will hereafter be referred to as DRP_Gar.

All considered dERC and DRP values are summarized in Table 1 . In the validation of the methods, using simulated data as described next, different com- 
Table 1. Description of the different computed variables that were considered as pseudo-phenotypes or weights thereof

\begin{tabular}{lll}
\hline Variable type & Abbreviation & Description \\
\hline Pseudo-phenotype & EBV & Estimated breeding value \\
Pseudo-phenotype & DRP_Gar & DRP ${ }^{1}$ obtained by deregressing Mendelian sampling term based on the reliability \\
Pseudo-phenotype & DRP_VR & DRP obtained by deregressing Mendelian sampling term based on the ratio between ERC \\
& & values with and without including parent information \\
Pseudo-phenotype & DRP_mat & DRP obtained with matrix deregression \\
Weight & ERC & Effective record contribution \\
Weight & dERC_VR & ERC corrected for information of parents included in the reference population \\
Weight & dERC_LHS & dERC ${ }^{2}$ computed from inverse of the left-hand side of the mixed model equations \\
Weight & dERC_iter & dERC computed iteratively ignoring inbreeding \\
Weight & dERC_iter_F & dERC computed iteratively considering inbreeding \\
\hline
\end{tabular}

${ }^{1}$ Deregressed proof.

${ }^{2}$ Deregressed effective record contribution (ERC).

binations of types of DRP and dERC were considered, where within combination the chosen dERC was computed first and always used both in the computations of the DRP and the computation of the final EBV. The most naive combination used EBV as DRP and ERC as dERC. Both the DRP_VR and DRP_Gar were computed together with either the ERC, dERC_VR, or dERC_LHS as dERC. Finally, the DRP_mat were combined with all 5 considered types of dERC.

\section{Validation of Deregression Procedures}

To validate the deregression procedures phenotypic data were simulated through an existing pedigree of cows and bulls. For simplicity, it was assumed that the repeatability of the trait was equal to the heritability (i.e., no permanent environmental effect was assumed). The simulated trait had either a heritability of 0.05 or 0.3 . The data contained 1,532 bulls and 13,720 cows that were considered to be included in the reference population, with a pedigree file of 35,005 animals in total. Each of the cows received 1 to 5 records with equal probability. For the cows, true dERC reflected the amount of own information, and were therefore equal to the number of records that a cow received. For the bulls, on top of the daughters already included in the data, additional daughters were simulated to reflect the part of their information that came from outside the reference population. Each bull received 50 to 200 additional effective daughters with equal probability, and each of those additional daughters had a single record (as defined by VanRaden and Wiggans, 1991). The ERC based on those additional daughters was the true ERC for the DRP, in the sense that it relates to the "own" information of the bulls that does not come from any (genotyped) animals inside the reference population. These true ERC were calculated for each bull as (Garrick et al., 2009):

$$
\mathrm{ERC}_{\text {true }}=n_{\mathrm{dtr}} \frac{1-h^{2}}{4-h^{2}},
$$

where $n_{\text {dtr }}$ is the additional number of effective daughters. So, considering the 50 to 200 additional daughters, the true ERC ranged from 12.0 to 48.1 for a heritability of 0.05 , and from 9.5 to 37.8 for a heritability of 0.30 .

The following analyses were performed on this simulated data:

1. EBV and ERC were estimated from the phenotypic data.

2. Obtained EBV and ERC were deregressed using the procedures outlined previously.

3. The DRP and dERC obtained in step 2 were used to estimate final EBV and ERC.

Step 1 was performed with ASReml (Gilmour et al., 2009), fixing the variances to the simulated values, where the reliability of animal $k$ was computed from the prediction error variance (PEV), as (Mrode, 2005): $\mathrm{REL}_{k}=1-\frac{\mathrm{PEV}_{k}}{A_{k k} \sigma_{a}^{2}}$, where $A_{k k}=1+F_{k}, F_{k}$ is the inbreeding coefficient of animal $k$, and $\sigma_{a}^{2}$ is the simulated genetic variance. Those reliabilities were subsequently used to compute the ERC values. Step 2 was performed with the procedures that were outlined previously (i.e., by first computing the dERC using the 5 proposed methods), and then computing DRP_VR and DRP mat using each of the 5 types of dERC. In step 2, any deregressed ERC with a value smaller than 1 were set to zero, before computing the DRP, such that those records were effectively removed. This was done because it is commonly known that deregression of EBV with (very) low reliability may lead to spurious results (Sigurdsson and Banos, 1995), whereas these records add very little information to the reference population. 
Step 3 tested the reversibility of the deregression by recalculating EBV from the DRP (Mäntysaari et al., 2011). This was performed with a straightforward BLUP animal model using the MiXBLUP software (Napel et al., 2014). In all 3 steps, the simulated variance components were used.

The validation of the deregression procedures involved comparison of the dERC values obtained in step 2 to the true (simulated) ERC that for the bulls were based on daughters outside the reference population, and for the cows based on their own records, as explained previously. The obtained dERC values were also validated by comparing the final ERC obtained in step 3 to the initial ERC obtained in step 1, where the expectation is that these are the same if the deregression procedures are $100 \%$ accurate. The final ERC were computed from the PEV of the model used to compute the final EBV. Note that this does not involve the DRP used, so those results for the final ERC are not affected by the method used to compute the DRP.

In a similar fashion, the final EBV from step 3 were compared with the initial EBV from step 1. For both using EBV and DRP_VR as DRP this can be regarded as a formal validation, because both are expected to yield final EBV that are similar to but not necessarily exactly the same as the initial EBV. For DRP_mat, provided that the values have converged properly, by definition the final EBV are expected to be exactly the same as the initial EBV, and only rounding errors of the DRP or dERC values could cause very small deviations.
Note that this validation of $\mathrm{dERC}$ and approximate DRP (i.e., regressed EBV and DRP_VR) is a straightforward test that can be performed for real data as well, whereas the comparison of the dERC values to the true ERC values is not possible in practice. The simulations and analyses as outlined above were replicated 20 times for heritabilities of 0.05 and 0.30 .

\section{RESULTS}

\section{Deregressed ERC}

Five different values were used as dERC value (i.e., as weight used to compute the DRP), but also as the weight of the DRP in the final BLUP analysis. Statistics of the differences between these dERC and the true ERC values are in Table 2 for the bulls and in Table 3 for the cows. For both the bulls and the cows, on average the ERC value of the EBV before deregression differed most from the true ERC (tERC). The difference between ERC and tERC is simply due to the fact that ERC also include contributions due to relationships, whereas tERC include only contributions due to "own" records. The value of dERC_LHS on average differed least from the true ERC. For 3 out of 4 scenarios, the next best dERC value was obtained by dERC_iter_F, which slightly outperformed dERC_iter, indicating that including the inbreeding coefficient in the approximate procedure was indeed beneficial. Finally, the dERC_VR value was closest to the (initial) ERC for the bulls. This observation could be expected because,

Table 2. Statistics of the differences between the simulated $\mathrm{ERC}^{1}$ (tERC) and $\mathrm{dERC}^{1}$ values of bulls, averaged across 20 replicates

\begin{tabular}{|c|c|c|c|c|c|c|c|}
\hline \multirow[b]{2}{*}{$h^{2}$} & \multirow[b]{2}{*}{$\mathrm{dERC}^{1}$} & \multicolumn{6}{|c|}{$(\mathrm{dERC}-\mathrm{tERC})$} \\
\hline & & $\mathrm{MSE}^{2}$ & Mean & Mean $(\mathrm{Abs})^{3}$ & Median & Minimum & Maximum \\
\hline \multirow[t]{5}{*}{0.3} & ERC & 485.29 & 8.87 & 8.87 & 3.73 & 1.17 & 329.21 \\
\hline & dERC_VR & 477.70 & 8.35 & 8.35 & 3.13 & 0.62 & 329.21 \\
\hline & dERC_LHS & 88.79 & 3.25 & 3.25 & 1.50 & -0.08 & 191.68 \\
\hline & dERC_iter & 156.29 & 4.68 & 4.68 & 2.42 & 0.04 & 243.86 \\
\hline & dERC_iter_F & 125.63 & 4.04 & 4.07 & 2.17 & -5.02 & 235.27 \\
\hline \multirow{5}{*}{0.05} & $\mathrm{ERC}$ & $2,769.93$ & 25.25 & 25.25 & 14.33 & 5.15 & 712.4 \\
\hline & dERC_VR & $2,621.99$ & 21.61 & 21.61 & 10.76 & 0.81 & 712.4 \\
\hline & dERC_LHS & 159.86 & 3.84 & 5.04 & 2.56 & -29.05 & 282.0 \\
\hline & dERC_iter & 403.26 & 8.44 & 11.85 & 8.80 & -47.44 & 408.6 \\
\hline & dERC_iter_F & 395.93 & 7.52 & 12.10 & 8.89 & -47.63 & 415.0 \\
\hline
\end{tabular}

${ }^{1} \mathrm{ERC}$ is the effective record contribution (ERC) of the EBV; dERC is the deregressed ERC; dERC_VR is the ERC corrected for information of parents in the reference population; dERC_LHS is the dERC computed based on inversion of the left-hand side of the corresponding mixed model equations; dERC_iter is the dERC computed by iteratively correcting for information of relatives in the reference population; dERC_iter_F is the same as dERC_iter but accounts for inbreeding.

${ }^{2}$ Mean squared error of the dERC value.

${ }^{3}$ Mean of absolute difference. 
dERC_VR tends to ERC when ERC increases, and high ERC are expected for bulls. For the cows, dERC VR was closest to dERC_iter, and actually seemed to outperform dERC_iter and dERC_iter_F for the cows and the trait with a heritability of 0.05 .

Interestingly, the median of the differences between dERC and true ERC was relatively close for cows and bulls. The minimum value of the differences was close to zero for both bulls and cows for the trait with a heritability of 0.30. Only for dERC_iter_F the minimum value became substantially lower than zero, indicating that dERC_iter_F values at least in some cases were considerably smaller than the true ERC values, whereas most other dERC values generally were greater than the true ERC values. For the heritability of 0.05 , the minimum differences for dERC_VR were still positive or very close to zero, indicating that almost all dERC_VR values overestimated the true ERC value.

The maximum value of the differences was clearly lowest for dERC_LHS, and ranking of the methods based on the maximum difference was generally similar to ranking of the methods based on increasing mean difference. Both the mean and the maximum values of the differences showed that for all methods the dERC values on average were overestimated. To further illustrate this, boxplots of the 5 different types of dERC values are shown in Figure 1 for each of the values of the true ERC values (1-5) for the cows for the trait with a heritability of 0.05 . This shows that the overestimation of the true ERC values indeed was lowest for dERC_LHS, and that the largest overestimation of dERC_iter and dERC_iter_F was smaller than for ERC and dERC_VR, although in this particular case
dERC_VR yielded an average closest to the true average. For the bulls, the same ranking of methods in terms of largest overestimation was observed (Figure 2).

Statistics of the comparison of final to initial ERC values, for each of the considered dERC values, are given in Table 4 for the bulls and in Table 5 for the cows. In all cases, dERC_LHS yielded final ERC values that were very close to the initial ones. The largest observed differences for dERC_LHS and the low heritability trait were generally for animals with a high ERC value. For the bulls, the dERC_iter_F yielded the second closest results. For the cows, there was not a clear second best method. For the low heritability trait on average the final ERC obtained from dERC_iter and dERC_iter_F overestimated the initial ERC, whereas those were on average underestimated when using dERC_VR.

\section{$D R P$}

Three different values were used as the DRP value, and these DRP values were computed using different dERC values. Subsequently, each considered combination of DRP and dERC values was used to compute final EBV. Statistics of the comparison of those final EBV to the initial EBV are in Table 6 for the bulls and in Table 7 for the cows. For both bulls and the cows, regardless of the dERC value used, EBV computed from DRP_mat were indeed very similar to the original EBV as expected. Although the correlation of the final to initial EBV for the bulls was only slightly lower than 1 when using EBV or DRP_VR as response variable, in both cases the variance of the final EBV was under-

Table 3. Statistics of the differences between the simulated $\mathrm{ERC}^{1}$ (tERC) and $\mathrm{dERC}^{1}$ values of cows, averaged across 20 replicates

\begin{tabular}{|c|c|c|c|c|c|c|c|}
\hline \multirow[b]{2}{*}{$h^{2}$} & \multirow[b]{2}{*}{$\mathrm{dERC}^{1}$} & \multicolumn{6}{|c|}{$(\mathrm{dERC}-\mathrm{tERC})$} \\
\hline & & $\mathrm{MSE}^{2}$ & Mean & Mean $(\mathrm{Abs})^{3}$ & Median & Minimum & Maximum \\
\hline \multirow[t]{5}{*}{0.3} & ERC & 4.28 & 2.03 & 2.03 & 1.99 & 0.00 & 12.66 \\
\hline & dERC_VR & 2.01 & 1.33 & 1.33 & 1.32 & -0.05 & 11.91 \\
\hline & dERC_LHS & 0.47 & 0.62 & 0.62 & 0.61 & -1.00 & 2.79 \\
\hline & dERC_iter & 1.87 & 1.29 & 1.29 & 1.31 & -1.00 & 4.63 \\
\hline & dERC_iter_F & 1.59 & 1.13 & 1.18 & 1.16 & -2.32 & 3.80 \\
\hline \multirow[t]{5}{*}{0.05} & ERC & 114.60 & 10.51 & 10.51 & 10.41 & -0.01 & 48.79 \\
\hline & dERC_VR & 42.77 & 6.00 & 6.00 & 5.78 & -0.19 & 43.20 \\
\hline & dERC_LHS & 4.39 & 1.77 & 1.80 & 1.63 & -3.69 & 12.32 \\
\hline & dERC_iter & 75.02 & 7.93 & 7.95 & 8.16 & -4.80 & 21.60 \\
\hline & dERC_iter_F & 78.95 & 8.00 & 8.07 & 8.90 & -5.00 & 20.56 \\
\hline
\end{tabular}

${ }^{1}$ ERC is the effective record contribution (ERC) of the EBV; dERC is the deregressed ERC; dERC_VR is the ERC corrected for information of parents in the reference population; dERC_LHS is the dERC computed based on inversion of the left-hand side of the corresponding mixed model equations; dERC_iter is the dERC computed by iteratively correcting for information of relatives in the reference population; dERC_iter_F is the same as dERC_iter but accounts for inbreeding.

${ }^{2}$ Mean squared error of the dERC value.

${ }^{3}$ Mean of absolute difference. 


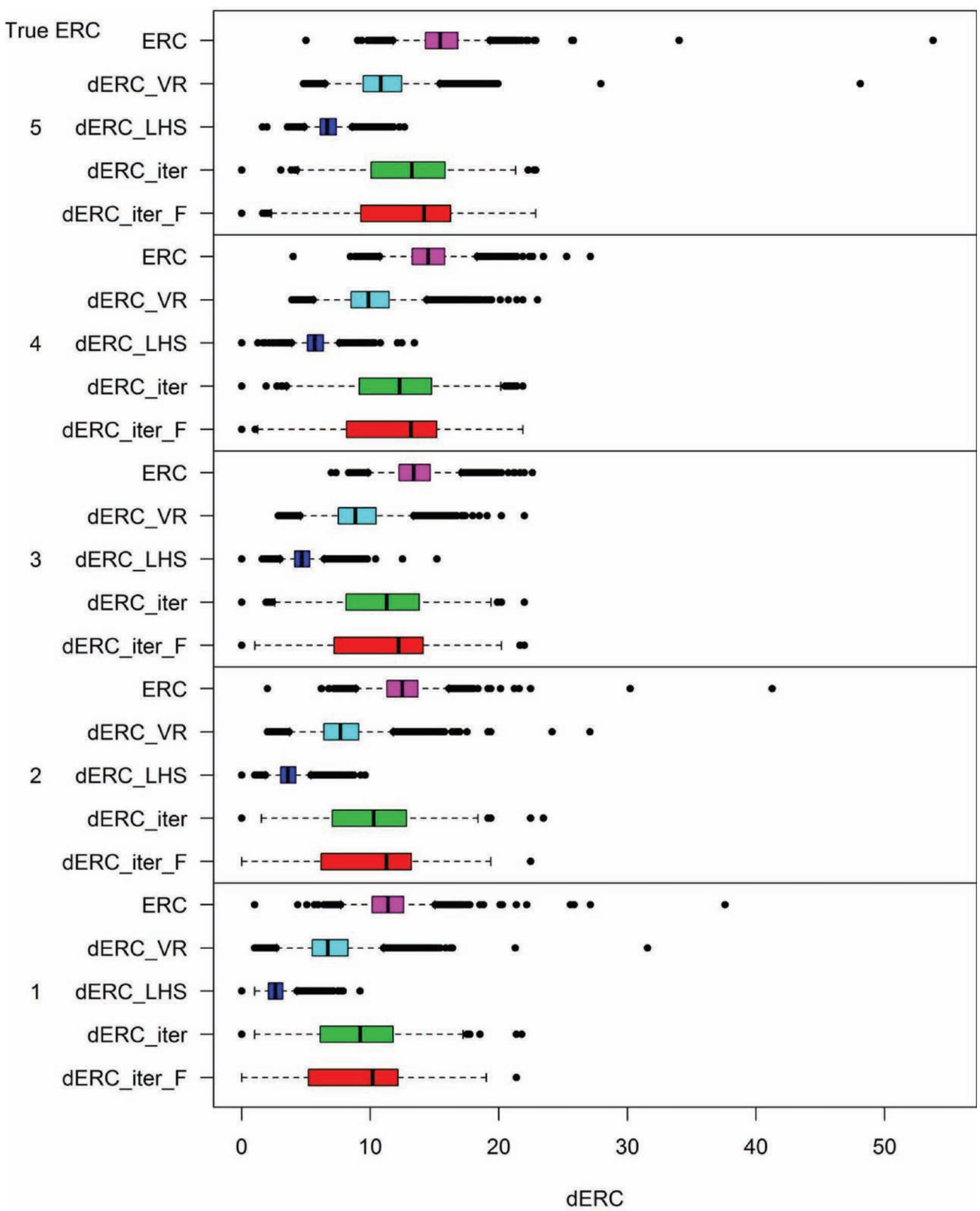

Figure 1. Simulated (true) versus deregressed effective record contribution (ERC) values for cows and the trait with a heritability of 0.05 , for the first replicate. Deregressed ERC were computed with 5 different methods: dERC is the deregressed ERC; dERC_VR is the ERC corrected for information of parents in the reference population; dERC_LHS is the dERC computed based on inversion of the left-hand side of the corresponding mixed model equations; dERC_iter is the dERC computed by iteratively correcting for information of relatives in the reference population; and dERC_iter_F is the same as dERC_iter but accounts for inbreeding. The box indicates the interquartile range; the vertical line in the box indicates the median; whiskers denote one time the interquartile range outside the box on either side; dots indicate outliers outside this range. Color version available online. 


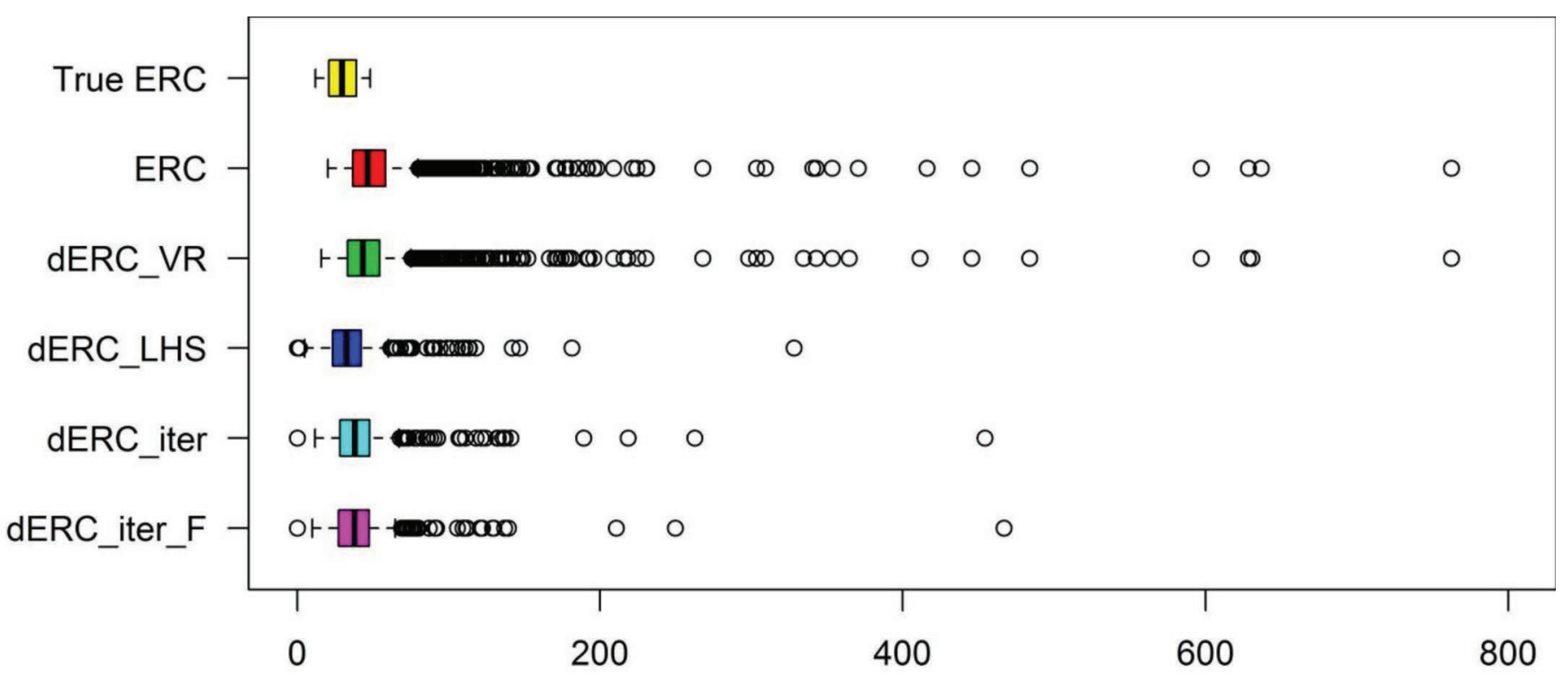

Figure 2. Simulated (true) and deregressed effective record contribution (ERC) values for bulls and the trait with a heritability of 0.05 , for the first replicate. Deregressed ERC were computed with 5 different methods: dERC is the deregressed ERC; dERC_VR is the ERC corrected for information of parents in the reference population; dERC_LHS is the dERC computed based on inversion of the left-hand side of the corresponding mixed model equations; dERC_iter is the dERC computed by iteratively correcting for information of relatives in the reference population; and dERC_iter_F is the same as dERC_iter but accounts for inbreeding. The box indicates the interquartile range; the vertical line in the box indicates the median; whiskers denote one time the interquartile range outside the box on either side; dots indicate outliers outside this range. Color version available online.

estimated, as shown by the regression coefficient being larger than 1. The variance of final EBV computed using DRP_VR combined with dERC_LHS, however, was inflated. The same patterns were observed for the cows, albeit that the differences between final EBV obtained from DRP_mat versus EBV and DRP_VR were more pronounced. When using DRP_Gar, in all cases the variance of the final EBV was inflated, especially for the final EBV of the cows for the low heritability trait. Generally, the deviation of the regression coefficients from the expected value of 1 was greater for the trait with the lower heritability.

Table 4. Statistics of the differences between the initial (iERC) and final ERC ${ }^{1}$ (fERC) values of bulls, depending on the $\mathrm{dERC}^{1}$ value used in the analysis, averaged across 20 replicates

\begin{tabular}{llrrrrrr}
\hline & & \multicolumn{7}{c}{$(\mathrm{fERC}-\mathrm{iERC})$} \\
\cline { 3 - 7 } $\mathrm{h}^{2}$ & & \multicolumn{7}{c}{ MSE $^{2}$} & Mean & Mean $(\mathrm{Abs})^{3}$ & Median & Minimum & Maximum \\
\hline 0.3 & dERC & & & & & \\
& ERC & 240.77 & 6.40 & 6.40 & 2.19 & 0.08 & 193.59 \\
& dERC_VR & 202.40 & 5.49 & 5.49 & 1.61 & 0.08 & 177.05 \\
& dERC_LHS & 0.00 & 0.00 & 0.00 & 0.00 & -0.26 & 0.00 \\
& dERC_iter & 16.84 & 1.78 & 1.78 & 1.05 & 0.08 & 60.96 \\
& dERC_iter_F & 5.44 & 0.54 & 1.09 & 0.52 & -20.78 & 38.76 \\
& ERC & 6045.95 & 33.45 & 33.45 & 13.92 & 0.48 & 976.7 \\
& dERC_VR & 533.39 & -20.65 & 20.65 & -18.81 & -184.01 & -12.6 \\
& dERC_LHS & 8.81 & -1.07 & 1.07 & -0.18 & -36.91 & 0.7 \\
& dERC_iter & 590.77 & 12.38 & 12.38 & 8.49 & 0.43 & 278.2 \\
& dERC_iter_F & 501.34 & 11.03 & 11.21 & 8.25 & -14.36 & 238.3 \\
\hline
\end{tabular}

${ }^{1} \mathrm{dERC}$ is the deregressed effective record contribution (ERC); dERC_VR is the ERC corrected for information of parents in the reference population; dERC_LHS is the dERC computed based on inversion of the left-hand side of the corresponding mixed model equations; dERC_iter is the dERC computed by iteratively correcting for information of relatives in the reference population; dERC_iter_F is the same as dERC_iter but accounts for inbreeding.

${ }^{2}$ Mean squared error of the final ERC value.

${ }^{3}$ Mean of absolute difference. 
Table 5. Statistics of the differences between the initial (iERC) and final ERC ${ }^{1}$ (fERC) values of cows, depending on the $\mathrm{dERC}^{1}$ value used in the analysis, averaged across 20 replicates

\begin{tabular}{llrrrrrr}
\hline & & \multicolumn{7}{c}{$(\mathrm{fERC}-\mathrm{iERC})$} \\
\cline { 3 - 7 } $\mathrm{h}^{2}$ & & & \multicolumn{7}{c}{} \\
\hline & dERC & MSE $^{2}$ & Mean & Mean $(\mathrm{Abs})^{3}$ & Median & Minimum & Maximum \\
\hline 0.3 & ERC & 2.42 & 1.48 & 1.48 & 1.41 & 0.00 & 13.49 \\
& dERC_VR & 0.73 & 0.75 & 0.75 & 0.70 & -0.72 & 11.56 \\
& dERC_LHS & 0.01 & 0.00 & 0.00 & 0.00 & -4.41 & 0.00 \\
& dERC_iter & 0.61 & 0.69 & 0.70 & 0.66 & -3.90 & 3.99 \\
& dERC_iter_F & 0.49 & 0.42 & 0.57 & 0.42 & -5.99 & 2.13 \\
0.05 & 112.25 & 10.19 & 10.19 & 10.08 & 0.00 & 79.45 \\
& ERC & 47.79 & -6.63 & 6.63 & -6.56 & -23.68 & -1.21 \\
& dERC_VR & 0.20 & -0.27 & 0.27 & -0.16 & -6.58 & 0.00 \\
& dERC_LHS & 59.83 & 6.90 & 6.90 & 6.78 & -0.72 & 22.22 \\
& dERC_iter & 64.48 & 6.93 & 7.01 & 7.98 & -7.51 & 19.36 \\
\hline
\end{tabular}

${ }^{1} \mathrm{dERC}$ is the deregressed effective record contribution (ERC); dERC_VR is the ERC corrected for information of parents in the reference population; dERC_LHS is the dERC computed based on inversion of the left-hand side of the corresponding mixed model equations; dERC_iter is the dERC computed by iteratively correcting for information of relatives in the reference population; dERC_iter_F is the same as dERC_iter but accounts for inbreeding.

${ }^{2}$ Mean squared error of the final ERC value.

${ }^{3}$ Mean of absolute difference.

\section{Convergence and Computation Time}

Computation time of dERC_VR was negligible. Computation of dERC_iter and dERC_iter_F gener- ally converged in 10 to 20 iterations, and only in a few situations used the maximum number of 100 iterations. Even when 100 iterations were used, computation time was only $\sim 1.5 \mathrm{~s}$. Computation of dERC_LHS always

Table 6. Averages and SE across 20 replicates of correlations $(\bar{r})$ between initial and final EBV of bulls, and the slope $(\bar{b})$ and intercept $(\bar{a})$ of the regression of initial on final EBV

\begin{tabular}{|c|c|c|c|c|c|c|c|c|}
\hline$h^{2}$ & $\mathrm{dERC}^{1}$ & $\mathrm{DRP}^{2}$ & $\bar{r}$ & $\bar{a}$ & $\bar{b}$ & $\mathrm{SE}(\bar{r})$ & $\operatorname{SE}(\bar{a})$ & $\mathrm{SE}(\bar{b})$ \\
\hline \multirow[t]{12}{*}{0.3} & ERC & EBV & 0.997 & 0.017 & 1.064 & 0.000 & 0.008 & 0.001 \\
\hline & $\mathrm{ERC}$ & DRP_Gar & 0.997 & 0.002 & 0.989 & 0.000 & 0.002 & 0.001 \\
\hline & dERC_VR & DRP_Gar & 0.997 & 0.019 & 0.988 & 0.000 & 0.013 & 0.001 \\
\hline & dERC_LHS & DRP_Gar & 0.996 & 0.021 & 0.981 & 0.000 & 0.013 & 0.002 \\
\hline & ERC & DRP_VR & 0.998 & 0.017 & 1.048 & 0.000 & 0.009 & 0.001 \\
\hline & dERC_VR & DRP_VR & 0.998 & 0.017 & 1.050 & 0.000 & 0.009 & 0.001 \\
\hline & dERC_LHS & DRP_VR & 0.989 & 0.025 & 0.931 & 0.000 & 0.010 & 0.002 \\
\hline & ERC & DRP_mat & 1.000 & 0.027 & 0.999 & 0.000 & 0.013 & 0.000 \\
\hline & dERC_VR & DRP_mat & 1.000 & 0.029 & 0.999 & 0.000 & 0.014 & 0.000 \\
\hline & dERC_LHS & DRP_mat & 1.000 & 0.030 & 0.998 & 0.000 & 0.015 & 0.000 \\
\hline & dERC_iter & DRP_mat & 1.000 & 0.029 & 0.998 & 0.000 & 0.014 & 0.000 \\
\hline & dERC_iter_F & DRP_mat & 1.000 & 0.029 & 0.998 & 0.000 & 0.014 & 0.000 \\
\hline \multirow[t]{12}{*}{0.05} & ERC & EBV & 0.981 & 0.002 & 1.179 & 0.000 & 0.003 & 0.005 \\
\hline & ERC & DRP_Gar & 0.977 & 0.005 & 0.879 & 0.001 & 0.003 & 0.004 \\
\hline & dERC_VR & DRP_Gar & 0.972 & -0.005 & 0.856 & 0.001 & 0.007 & 0.004 \\
\hline & dERC_LHS & DRP_Gar & 0.941 & 0.001 & 0.728 & 0.002 & 0.007 & 0.006 \\
\hline & $\mathrm{ERC}$ & DRP_VR & 0.982 & 0.001 & 1.108 & 0.000 & 0.004 & 0.004 \\
\hline & dERC_VR & DRP_VR & 0.980 & 0.001 & 1.129 & 0.000 & 0.004 & 0.005 \\
\hline & dERC_LHS & DRP_VR & 0.937 & 0.010 & 0.833 & 0.006 & 0.005 & 0.026 \\
\hline & ERC & DRP_mat & 0.999 & 0.024 & 0.994 & 0.000 & 0.011 & 0.000 \\
\hline & dERC_VR & DRP_mat & 0.997 & 0.029 & 0.979 & 0.002 & 0.013 & 0.015 \\
\hline & dERC_LHS & DRP_mat & 0.999 & 0.034 & 0.992 & 0.000 & 0.016 & 0.001 \\
\hline & dERC_iter & DRP_mat & 0.999 & 0.027 & 0.995 & 0.000 & 0.012 & 0.001 \\
\hline & dERC_iter_F & DRP_mat & 0.999 & 0.027 & 0.995 & 0.000 & 0.012 & 0.001 \\
\hline
\end{tabular}

${ }^{1} \mathrm{dERC}$ is the deregressed effective record contribution (ERC); dERC_VR is the ERC corrected for information of parents in the reference population; dERC_LHS is the dERC computed based on inversion of the left-hand side of the corresponding mixed model equations; dERC_iter is the dERC computed by iteratively correcting for information of relatives in the reference population; dERC_iter_F is the same as dERC_iter but accounts for inbreeding.

${ }^{2} \mathrm{DRP}$ is deregressed proof; EBV is the initial EBV; DRP_Gar is obtained by deregressing the Mendelian sampling term based on the reliability; DRP_VR is obtained by deregressing the Mendelian sampling term based on the ratio between ERC values with and without including parent information; DRP_mat is obtained with matrix deregression. 
used the maximum allowed number of 5 iterations, which took slightly more than $10 \mathrm{~min}$, using 4 cores to do the matrix inversions. It should be noted that in this case 6 matrices needed to be inverted with the dimension of $n \times n$ or $(n+1) \times(n+1)$, where $n$ is the number of animals whose EBV needed to be deregressed. This included the A matrix once and 5 times, once per iteration, the left-hand side of the MME of the model.

Computation time of the procedure to compute DRP_VR was negligible. Values for DRP_mat converged in all replicates in 3 to 5 iterations for the trait with a heritability of 0.3 . For the low heritability trait on average 25.9 iterations were required. One iteration required $\sim 30 \mathrm{~s}$, so convergence took anywhere between $\sim 2$ and 45 min.

\section{DISCUSSION}

The objective of this paper was to compare the performance of different deregression procedures for data that includes both cow and bull EBV, and to develop and test procedures to obtain the appropriate dere- gressed weights that should be used with those DRP. Although targeting a situation with cow and bull EBV, the methods presented are not only applicable to the dairy context, but can generally be used in any data structure including phenotypes of related individuals. As expected, DRP_mat yielded final EBV that were virtually the same as the original ones, regardless of which estimator was used for dERC. The other considered DRP did yield biased results, whereas the correlation between initial and final EBV was lower than 1, with the lowest value observed for the DRP of the cows for the low heritability trait. The reduced variance when using EBV or DRP_VR as response variable, suggest that the variance of both response variables is too low, indicating that the deregression performed with DRP_VR is too modest. Comparing the factor $\frac{\mathrm{ERC}_{k}}{\mathrm{dERC}_{k}}$ that is used to compute DRP_VR to the factor $\frac{1}{\operatorname{REL}_{k}(o+p)}$ used in DRP_Gar confirmed that the deregression performed for DRP_Gar is much stronger than for DRP_VR (results not shown). In fact, for DRP_Gar the variance of the final EBV was too large,

Table 7. Averages and SE across 20 replicates of correlations $(\bar{r})$ between initial and final EBV of cows, and the slope $(\bar{b})$ and intercept $(\bar{a})$ of the regression of initial on final EBV

\begin{tabular}{|c|c|c|c|c|c|c|c|c|}
\hline$h^{2}$ & $\mathrm{dERC}^{1}$ & $\mathrm{DRP}^{2}$ & $\bar{r}$ & $\bar{a}$ & $\bar{b}$ & $\mathrm{SE}(\bar{r})$ & $\mathrm{SE}(\bar{a})$ & $\mathrm{SE}(\bar{b})$ \\
\hline \multirow[t]{12}{*}{0.3} & ERC & EBV & 0.973 & 0.020 & 1.173 & 0.000 & 0.009 & 0.004 \\
\hline & ERC & DRP_Gar & 0.982 & 0.003 & 0.942 & 0.000 & 0.004 & 0.003 \\
\hline & dERC_VR & DRP_Gar & 0.979 & 0.020 & 0.936 & 0.000 & 0.011 & 0.003 \\
\hline & dERC_LHS & DRP_Gar & 0.971 & 0.021 & 0.906 & 0.000 & 0.011 & 0.004 \\
\hline & ERC & DRP_VR & 0.981 & 0.019 & 1.106 & 0.000 & 0.009 & 0.003 \\
\hline & dERC_VR & DRP_VR & 0.976 & 0.020 & 1.125 & 0.000 & 0.009 & 0.004 \\
\hline & dERC_LHS & DRP_VR & 0.963 & 0.027 & 0.943 & 0.000 & 0.013 & 0.004 \\
\hline & $\mathrm{ERC}$ & DRP_mat & 1.000 & 0.027 & 0.995 & 0.000 & 0.013 & 0.000 \\
\hline & dERC_VR & DRP_mat & 1.000 & 0.028 & 0.994 & 0.000 & 0.013 & 0.000 \\
\hline & dERC_LHS & DRP_mat & 1.000 & 0.029 & 0.993 & 0.000 & 0.014 & 0.000 \\
\hline & dERC_iter & DRP_mat & 1.000 & 0.028 & 0.994 & 0.000 & 0.013 & 0.000 \\
\hline & dERC_iter_F & DRP_mat & 0.999 & 0.027 & 0.996 & 0.000 & 0.012 & 0.001 \\
\hline \multirow[t]{12}{*}{0.05} & ERC & EBV & 0.955 & 0.001 & 1.139 & 0.001 & 0.003 & 0.005 \\
\hline & ERC & DRP_Gar & 0.947 & 0.002 & 0.726 & 0.001 & 0.001 & 0.005 \\
\hline & dERC_VR & DRP_Gar & 0.938 & -0.005 & 0.683 & 0.001 & 0.006 & 0.005 \\
\hline & dERC_LHS & DRP_Gar & 0.888 & -0.001 & 0.510 & 0.002 & 0.005 & 0.006 \\
\hline & ERC & DRP_VR & 0.965 & 0.001 & 1.014 & 0.001 & 0.003 & 0.005 \\
\hline & dERC_VR & DRP_VR & 0.953 & 0.001 & 1.054 & 0.001 & 0.003 & 0.006 \\
\hline & dERC_LHS & DRP_VR & 0.826 & 0.004 & 0.576 & 0.058 & 0.005 & 0.039 \\
\hline & $\mathrm{ERC}$ & DRP_mat & 0.999 & 0.023 & 0.991 & 0.000 & 0.011 & 0.002 \\
\hline & dERC_VR & DRP_mat & 0.989 & 0.028 & 0.981 & 0.009 & 0.012 & 0.008 \\
\hline & dERC_LHS & DRP_mat & 0.997 & 0.032 & 0.984 & 0.001 & 0.015 & 0.004 \\
\hline & dERC_iter & DRP_mat & 0.998 & 0.025 & 0.989 & 0.001 & 0.012 & 0.002 \\
\hline & dERC_iter_F & DRP_mat & 0.998 & 0.025 & 0.989 & 0.001 & 0.011 & 0.002 \\
\hline
\end{tabular}

${ }^{1}$ dERC is the deregressed effective record contribution (ERC); dERC_VR is the ERC corrected for information of parents in the reference population; dERC_LHS is the dERC computed based on inversion of the left-hand side of the corresponding mixed model equations; dERC_iter is the dERC computed by iteratively correcting for information of relatives in the reference population; dERC_iter_F is the same as dERC_iter but accounts for inbreeding.

${ }^{2} \mathrm{DRP}$ is the deregressed proof; EBV is the initial EBV; DRP_Gar is obtained by deregressing the Mendelian sampling term based on the reliability; DRP_VR is obtained by deregressing the Mendelian sampling term based on the ratio between ERC values with and without including parent information; DRP_mat is obtained with matrix deregression. 
suggesting that in this case the deregression was too strong. It should be noted that final EBV based on DRP_VR or DRP_Gar are both prone to doublecounting, because both DRP are obtained by only adjusting for PA and not, for example, for EBV of progeny whose EBV are also being deregressed. Apparently, the too modest deregression in DRP_VR coincidently partly counteracts the double-counting. This is not the case for the much stronger deregression in DRP_Gar, despite the notion that the above mentioned factor in the DRP_Gar deregression in itself seems more appropriate. Our results for the cows especially seem to be in line with the results of a study on pigs with relatively low accuracy EBV that using appropriate DRP yielded more reliable genomic predictions than using EBV, albeit that the effect on bias of the genomic predictions was not reported (Ostersen et al., 2011).

The importance of deregression, including accounting for family information, is expected to be higher for low compared with high heritability traits (Mark et al., 2002), simply because family information is relatively more important for low heritability traits. In the context of validation of genomic prediction, it has been shown that use of DRP derived from evaluations including information of both reference and validation animals leads to overestimation of the accuracy. This overestimation was found to be higher for low compared with high heritability traits (Amer and Banos, 2010). Considering that genomic evaluations are in several cases relying on DRP, the observed tendency that medium to high heritability traits more easily pass the GEBV Interbull test than low heritability traits (Loberg et al., 2011) may also be due to the use of suboptimal deregression procedures. In summary, these expectations and results from the literature are in line with our results that showed that use of suboptimal deregression procedures has the largest effect on final EBV of low heritability traits.

The simulated data used in our study was relatively simplistic, in the sense that, for example, no structure of contemporary groups or other fixed effects was simulated. Presence of contemporary groups could induce covariances between ERC of cows in the same group. The information of such structure, however, in many cases may not be available when performing the deregression. As a consequence, in the deregression commonly the only considered fixed effect is a mean as in our study and other studies (e.g., Jairath et al., 1998; Strandén and Mäntysaari, 2010), or fixed effects are simply not considered in the deregression step (e.g., Lien et al., 1995; Harris and Johnson, 2010). Only including a mean and further ignoring fixed effects structures in the procedures to compute DRP and their weights was, however, shown to hardly affect the final EBV of bulls, provided that the DRP are properly corrected for information from relatives included in the final genetic evaluation system (Mäntysaari et al., 2011; Vandenplas and Gengler, 2012). This is in line with our observation that when using the most optimal procedure to compute DRP (i.e., DRP_mat), the final EBV were very close to the initial EBV, both for bulls and cows, regardless of the type of weights used for the DRP.

\section{Deregression of ERC}

The method to compute dERC_LHS was theoretically expected to be the most optimal for the following 2 reasons. First, it directly attempts to compute dERC values that would yield ERC in the subsequent BLUP analysis that are similar to the initial ERC. Second, it uses inversion of the left-hand side of the MME, and thereby considers both prediction error variances and covariances, whereas, for example, dERC_iter and dERC_iter F only considered prediction error variances. Our results confirmed that the dERC_LHS yielded final ERC values very close to initial ERC values, whereas it showed on average small deviations from the true ERC values. In fact, all methods generally tended to overestimate the true ERC values. This indicates that the methods used simply are not well enough able to further distinguish between own information and information from relatives. Nevertheless, despite this general overestimation of dERC values, the final ERC values may still be almost identical to the initial ERC, as demonstrated by the results based on dERC_LHS.

The literature shows some support that using appropriate weights in similar analyses, rather than assuming the same weight for each animal, improves accuracy of genomic prediction (Villumsen et al., 2009; Guo et al., 2010) and also for QTL mapping (Thomsen et al., 2001). Combining dERC_LHS and DRP_VR yielded a clear mismatch in our study, as the variance of the resulting final EBV was severely overestimated. This probably occurred because the dERC_LHS values too strongly inflated the mendelian sampling term in the process of computing the DRP_VR values. It should be noted that the final EBV obtained from DRP_mat values should be the same as the initial values, regardless of which type of dERC values are used, and therefore these results are not informative to draw any conclusions related to the effect of the weights used on the final EBV.

In applications where the final ERC values are used to compute individual reliabilities, either based on PEV obtained from the inverted LHS, or based on an approximation approach (e.g., Misztal and Wiggans, 1988; VanRaden and Wiggans, 1991), it is however important that the dERC values indeed reflect the amount of 
"own" information retained in the DRP. Again, this is especially important for animals with low reliabilities. For instance, in a study on integration of different sources of information in genetic evaluation, it has been shown that double-counting of information on average inflated the reliabilities from 0.43 to 0.57 for animals with initial reliabilities $<0.5$, whereas the ranking of those animals was hardly affected (Vandenplas et al., 2014b). Based on our results it seems advisable to use either dERC_LHS or dERC_iter_F to avoid such inflation of the reliabilities. The dERC_LHS gave the best result, but the computation may become demanding if the number of animals in the reference population is much larger than in the data used in our study. In such cases, dERC_iter_F generally appears to be a proper alternative, as its computation requirements are trivial.

\section{Deregression Methods and Applications}

Two different deregression procedures were used in our study. Several other procedures have been proposed in the literature. One example is a deregression procedure specifically developed to combine own performances from genotyped individuals with performances of relatives, such as sibs, that are not genotyped (Ricard et al., 2013). Effectively, their method achieves the same as the matrix deregression procedure in our paper. As expected beforehand, and confirmed by our results, proper deregression is more important for animals with low initial reliabilities (e.g., cows) compared with bulls that generally have substantially larger reliabilities.

In this paper, we refer to a situation in which EBV of animals included in a reference population for genomic prediction need to be deregressed to be used as pseudo-phenotypes in a subsequent genomic prediction model. The described methods are also applicable for other situations. Indeed, deregression methods were initially used by methods developed to compare dairy sires evaluated in different countries (e.g., Goddard, 1985; Rozzi et al., 1990). Deregression methods were also explicitly applied to compute pseudo-phenotypes and associated weights to include foreign information (summarized, e.g., as EBV and REL) in national polygenetic genetic evaluations (e.g., Bonaiti and Boichard, 1995; VanRaden et al., 2014). The referred situation in this paper in which pseudo-phenotypes are considered in a genomic prediction model, is in fact very similar to a situation where foreign information is included in an evaluation whereas no local phenotypes are available. A combination of both local (phenotypes) and foreign information (as weighted pseudo-phenotypes) in a ssGBLUP was proposed by Prribyl et al. (2013) in which they used a matrix deregression method, whereas the weights did not take double-counting of contributions due to relationships into account. Other methods, such as absorption (Henderson, 1975) or Bayesian methods (e.g., Legarra et al., 2007; Vandenplas et al., 2014a), that are used to include foreign information in genetic or genomic evaluations can also benefit from our proposed approach to compute dERC free of information from relatives, considering that absorption, Bayesian, and pseudo-phenotype-based methods are, at least theoretically, equivalent (Bonaiti and Boichard, 1995; Vandenplas and Gengler, 2015).

Several studies also proposed to deregress direct genomic values and associated REL to combine them with conventional data (e.g., Ducrocq and Liu, 2009; Stoop et al., 2013). Double-counting of contributions due to relationships could be even worse in these cases because all genotyped animals could contribute to the information of all other genotyped animals, instead of only parents, progeny, or other relatives in a pedigreebased model. Our proposed method to compute dERC_LHS could be used to avoid this double-counting of contributions due to pedigree and genomic relationships by replacing $\mathbf{A}_{\mathrm{gg}}^{-1}$ by the inverse of a genomic relationship matrix $\left(\mathbf{G}^{-1}\right)$. This is straightforward for a simple GBLUP type of model that allocates equal variances to all SNP, but also possible for models that employ differential shrinkage, for instance by computing a weighted $\mathbf{G}$ based on the SNP variances estimated in the model (Zhang et al., 2010). If those SNP variances are not available, an unweighted $\mathbf{G}$ could be used as an approximation instead.

\section{Deregression in the Context of Genomic Prediction}

Although the methods evaluated in our study can be used for different applications, we particularly had the application for subsequent genomic prediction in mind. Further evaluation of the effect of the deregression procedure could involve performing an Interbull test (Mäntysaari et al., 2010) with real data on genotyped animals. This evaluation would focus on the effect on the obtained genomic EBV, their unbiasedness, and their reliabilities. As previously indicated, the effect of the type of dERC values used may be important for the computation of individual reliabilities, either based on $\mathrm{PEV}$ or approximation methods. If the reliabilities are computed using (cross-)validation, the effect is expected to be limited to the unbiasedness of the predictions.

\section{CONCLUSIONS}

This study described procedures to evaluate deregression methods, that can be applied on simulated data, and partly also on real data. Several methods to compute DRP and their weights were presented and 
used for simultaneous deregression of cow and bull EBV. The applicability of the methods presented, however, is not restricted to the dairy context, but more generally extends to other data structures including phenotypes with heterogeneous information content of related individuals. The methods developed to compute appropriate weights for the DRP were either very precise and computationally somewhat demanding for larger data sets (dERC_LHS), or were less precise but computationally trivial due their approximate nature (dERC_iter and dERC_iter_F). So, for small data sets using dERC_LHS is feasible, whereas for large data sets dERC_iter_F is much more convenient. Using the matrix deregression method, which yields by definition final EBV that are identical to the original EBV, is preferred over using a simpler approach that only corrected for information of parents or not performing any deregression at all. The type of weights used for DRP are important when either an approximate procedure is used to deregress the EBV, or when individual reliabilities of final EBV are computed based on the prediction error variance of the model. In those cases, the weights of the DRP should be properly corrected for information of relatives that is also included in the data, to avoid double-counting of information.

\section{ACKNOWLEDGMENTS}

The authors acknowledge CRV BV (Arnhem, the Netherlands) and the Dutch Ministry of Economic Affairs, Agriculture, and Innovation (public-private partnership "Breed4Food" code BO-22.04-011-001ASG-LR) for financial support.

\section{REFERENCES}

Amer, P. R., and G. Banos. 2010. Implications of avoiding overlap between training and testing data sets when evaluating genomic predictions of genetic merit. J. Dairy Sci. 93:3320-3330.

Anderson, E., Z. Bai, J. Dongarra, A. Greenbaum, A. McKenney, J. Du Croz, S. Hammerling, J. Demmel, C. Bischof, and D. Sorensen. 1990. LAPACK: A portable linear algebra library for high-performance computers. Pages 2-11 in Proc. 1990 ACM/IEEE Conference on Supercomputing. IEEE Computer Society Press, Los Alamitos, CA.

Banos, G., J. Philipsson, M. Gundel, J. Juga, and U. Sander-Nielsen. 1993. Linear model comparisons of black-and-white dairy bulls from the Nordic countries. Interbull Bull. 8:1-12.

Banos, G., G. R. Wiggans, and J. A. B. Robinson. 1992. Comparison of methods to rank bulls across countries. J. Dairy Sci. 75:2560-2568.

Boichard, D., C. Grohs, F. Bourgeois, F. Cerqueira, R. Faugeras, A. Neau, R. Rupp, Y. Amigues, M. Y. Boscher, and H. Levéziel. 2003. Detection of genes influencing economic traits in three French dairy cattle breeds. Genet. Sel. Evol. 35:77-101.

Bonaiti, B., and D. Boichard. 1995. Accounting for foreign information in genetic evaluation. Interbull Bulletin no. 11. Proc. of the Open Session of the Interbull Annual Meeting, Prague, Czech Republic.

Buch, L. H., M. Kargo, P. Berg, J. Lassen, and A. C. Sorensen. 2012. The value of cows in reference populations for genomic selection of new functional traits. Animal 6:880-886.
Calus, M. P. L., Y. de Haas, and R. F. Veerkamp. 2013. Combining cow and bull reference populations to increase accuracy of genomic prediction and genome-wide association studies. J. Dairy Sci. 96:6703-6715.

Colleau, J. J. 2002. An indirect approach to the extensive calculation of relationship coefficients. Genet. Sel. Evol. 34:409-421.

Ducrocq, V. 1994. Statistical-analysis of length of productive life for dairy-cows of the Normande breed. J. Dairy Sci. 77:855-866.

Ducrocq, V., and Z. Liu. 2009. Combining genomic and classical information in national BLUP evaluations. Interbull Bull. 40:172-176.

Egger-Danner, C., H. Schwarzenbacher, and A. Willam. 2014. Short communication: Genotyping of cows to speed up availability of genomic estimated breeding values for direct health traits in Austrian Fleckvieh (Simmental) cattle - Genetic and economic aspects. J. Dairy Sci. 97:4552-4556.

Fernando, R., J. Dekkers, and D. Garrick. 2014. A class of Bayesian methods to combine large numbers of genotyped and non-genotyped animals for whole-genome analyses. Genet. Sel. Evol. 46:50.

Fikse, W. F., and G. Banos. 2001. Weighting factors of sire daughter information in international genetic evaluations. J. Dairy Sci. 84:1759-1767.

Garrick, D. J., J. F. Taylor, and R. L. Fernando. 2009. Deregressing estimated breeding values and weighting information for genomic regression analyses. Genet. Sel. Evol. 41:55.

Gilmour, A. R., B. J. Gogel, B. R. Cullis, and R. Thompson. 2009 ASReml User Guide Release 3.0. VSN International Ltd., Hemel Hempstead, UK.

Goddard, M. 1985. A method of comparing sires evaluated in different countries. Livest. Prod. Sci. 13:321-331.

Guo, G., M. S. Lund, Y. Zhang, and G. Su. 2010. Comparison between genomic predictions using daughter yield deviation and conventional estimated breeding value as response variables. J. Anim. Breed. Genet. 127:423-432.

Hager, W. W. 1989. Updating the inverse of a matrix. SIAM Rev. $31: 221-239$

Harris, B., and D. Johnson. 1998. Approximate reliability of genetic evaluations under an animal model. J. Dairy Sci. 81:2723-2728.

Harris, B. L., and D. L. Johnson. 2010. Genomic predictions for New Zealand dairy bulls and integration with national genetic evaluation. J. Dairy Sci. 93:1243-1252.

Henderson, C. 1949. Estimation of changes in herd environment. J. Dairy Sci. 32:706.

Henderson, C. R. 1975. Use of all relatives in intraherd prediction of breeding values and producing abilities. J. Dairy Sci. 58:19101916.

Jairath, L., J. C. M. Dekkers, L. R. Schaeffer, Z. Liu, E. B. Burnside, and B. Kolstad. 1998. Genetic evaluation for herd life in Canada. J. Dairy Sci. 81:550-562.

Koivula, M., I. Strandén, G. P. Aamand, and E. A. Mäntysaari. 2016. Effect of cow reference group on validation reliability of genomic evaluation. Animal http://dx.doi.org/http://dx.doi.org/10.1017/ S1751731115002864.

Legarra, A., J. K. Bertrand, T. Strabel, R. L. Sapp, J. P. Sánchez, and I. Misztal. 2007. Multi-breed genetic evaluation in a Gelbvieh population. J. Anim. Breed. Genet. 124:286-295

Lien, S., L. Gomez-Raya, T. Steine, E. Fimland, and S. Rogne. 1995. Associations between casein haplotypes and milk yield traits. J. Dairy Sci. 78:2047-2056.

Loberg, A., H. Jorjani, and J. Dürr. 2011. Validation of genomic national evaluations. Interbull Bull. 44:62-66.

Lourenco, D. A. L., I. Misztal, S. Tsuruta, I. Aguilar, E. Ezra, M. Ron, A. Shirak, and J. I. Weller. 2014. Methods for genomic evaluation of a relatively small genotyped dairy population and effect of genotyped cow information in multiparity analyses. J. Dairy Sci. 97:1742-1752

Lund, M., S. de Roos, A. de Vries, T. Druet, V. Ducrocq, S. Fritz, F. Guillaume, B. Guldbrandtsen, Z. Liu, R. Reents, C. Schrooten, F Seefried, and G. Su. 2011. A common reference population from four European Holstein populations increases reliability of genomic predictions. Genet. Sel. Evol. 43:43. 
Mäntysaari, E., Z. Liu, and P. VanRaden. 2010. Interbull validation test for genomic evaluations. Interbull Bull. 41:17-22.

Mäntysaari, E. A., M. Koivula, I. Strandén, J. Pösö, and G. P. Aamand. 2011. Estimation of GEBVs using deregressed individual cow breeding values. Interbull Bull. 44:19-24.

Mark, T., W. F. Fikse, U. Emanuelson, and J. Philipsson. 2002. Short communication: Effect of phantom parent grouping and properties of deregression for a low heritability trait. J. Dairy Sci. 85:23932395.

Mc Hugh, N., T. H. E. Meuwissen, A. R. Cromie, and A. K. Sonesson. 2011. Use of female information in dairy cattle genomic breeding programs. J. Dairy Sci. 94:4109-4118.

Meuwissen, T. H. E., B. J. Hayes, and M. E. Goddard. 2001. Prediction of total genetic value using genome-wide dense marker maps. Genetics 157:1819-1829.

Misztal, I., and G. R. Wiggans. 1988. Approximation of prediction error variance in large-scale animal models. J. Dairy Sci. 71(Suppl. 2):27-32.

Mrode, R. 2005. Linear Models for the Prediction of Animal Breeding Values. 2nd ed. CABI Publishing.

Napel, J. t., H. A. Mulder, M. Lidauer, I. Stranden, E. Mäntysaari, M. H. Pool, and R. F. Veerkamp. 2014. MiXBLUP, the Mixed-model Best Linear Unbiased Prediction software for PCs for large genetic evaluation systems. Version 1.3.1., Wageningen, the Netherlands.

Ostersen, T., O. Christensen, M. Henryon, B. Nielsen, G. Su, and P. Madsen. 2011. Deregressed EBV as the response variable yield more reliable genomic predictions than traditional EBV in purebred pigs. Genet. Sel. Evol. 43:38.

Přibyl, J., P. Madsen, J. Bauer, J. Přibylová, M. Šimečková, L. Vostrý, and L. Zavadilová. 2013. Contribution of domestic production records, Interbull estimated breeding values, and single nucleotide polymorphism genetic markers to the single-step genomic evaluation of milk production. J. Dairy Sci. 96:1865-1873.

Ricard, A., S. Danvy, and A. Legarra. 2013. Computation of deregressed proofs for genomic selection when own phenotypes exist with an application in French show-jumping horses. J. Anim. Sci. 91:1076-1085.

Rodriguez-Zas, S. L., B. R. Southey, D. W. Heyen, and H. A. Lewin 2002. Interval and composite interval mapping of somatic cell score, yield, and components of milk in dairy cattle. J. Dairy Sci. 85:3081-3091.

Rogers, G., G. Banos, U. S. Nielsen, and J. Philipsson. 1996. Genetic correlations among somatic cell scores, productive life, and type traits from the United States and udder health measures from Denmark and Sweden. Interbull Bull. 14:34-38.

Rozzi, P., L. R. Schaeffer, E. B. Burnside, and W. Schlote. 1990. International evaluation of Holstein-Friesian dairy sires from three countries. Livest. Prod. Sci. 24:15-28.

Sargolzaei, M., H. Iwaisaki, and J. J. Colleau. 2005. A fast algorithm for computing inbreeding coefficients in large populations. J. Anim. Breed. Genet. 122:325-331.

Schaeffer, L. R. 1994. Multiple-country comparison of dairy sires. J. Dairy Sci. 77:2671-2678.
Shewchuck, J. 1994. Page 50 in An Introduction to the Conjugate Gradient Method Without the Agonizing Pain. Accessed Jan. 14, 2016. https://www.cs.cmu.edu/ quake-papers/painless-conjugategradient.pdf.

Sigurdsson, A., and G. Banos. 1995. Dependent-variables in international sire evaluations. Acta Agric. Scand. Anim. Sci. 45:209-217.

Stoop, M., H. Eding, M. L. van Pelt, L. C. M. de Haer, and G. de Jong. 2013. Using pseudo-observations to combine genomic and conventional data in the Dutch-Flemish national evaluation. Interbull Bull. 47:106-110.

Strandén, I., and E. A. Mäntysaari. 2010. A recipe for multiple trait deregression. Interbull Bull. 42:21-24.

Thomsen, H., N. Reinsch, N. Xu, C. Looft, S. Grupe, C. Kühn, G. A. Brockmann, M. Schwerin, B. Leyhe-Horn, S. Hiendleder, G. Erhardt, I. Medjugorac, I. Russ, M. Förster, B. Brenig, F. Reinhardt, R. Reents, J. Blümel, G. Averdunk, and E. Kalm. 2001. Comparison of estimated breeding values, daughter yield deviations and de-regressed proofs within a whole genome scan for QTL. J. Anim. Breed. Genet. 118:357-370.

Vandenplas, J., O. F. Christensen, and N. Gengler. 2014a. Short communication: Alteration of priors for random effects in Gaussian linear mixed models. J. Dairy Sci. 97:5880-5884.

Vandenplas, J., F. Colinet, and N. Gengler. 2014b. Unified method to integrate and blend several, potentially related, sources of information for genetic evaluation. Genet. Sel. Evol. 46:59.

Vandenplas, J., and N. Gengler. 2012. Comparison and improvements of different Bayesian procedures to integrate external information into genetic evaluations. J. Dairy Sci. 95:1513-1526.

Vandenplas, J., and N. Gengler. 2015. Strategies for comparing and combining different genetic and genomic evaluations: A review. Livest. Sci. 181:121-130.

VanRaden, P. M., C. P. Van Tassell, G. R. Wiggans, T. S. Sonstegard R. D. Schnabel, J. F. Taylor, and F. S. Schenkel. 2009. Invited review: Reliability of genomic predictions for North American Holstein bulls. J. Dairy Sci. 92:16-24.

VanRaden, P. M., and G. R. Wiggans. 1991. Derivation, calculation, and use of national animal-model information. J. Dairy Sci. 74:2737-2746.

VanRaden, P. M., M. E. Tooker, J. R. Wright, C. Sun, and J. L. Hutchison. 2014. Comparison of single-trait to multi-trait national evaluations for yield, health, and fertility. J. Dairy Sci. 97:79527962 .

Villumsen, T. M., L. Janss, P. Madsen, and M. Lund. 2009. EBV and DYD as response variable in genomic predictions. Page 299 in Book of Abstracts of the 60th Annual Meeting of the EAAP, Barcelona, Spain.

Wiggans, G. R., T. A. Cooper, P. M. VanRaden, and J. B. Cole. 2011. Technical note: Adjustment of traditional cow evaluations to improve accuracy of genomic predictions. J. Dairy Sci. 94:6188-6193.

Zhang, Z., J. F. Liu, X. D. Ding, P. Bijma, D. J. de Koning, and Q. Zhang. 2010. Best linear unbiased prediction of genomic breeding values using a trait-specific marker-derived relationship matrix. PLoS ONE 5:e12648. 